\title{
Soybean allergy in a population with a low prevalence of betulacae pollen allergy and a high soybean consumption
}

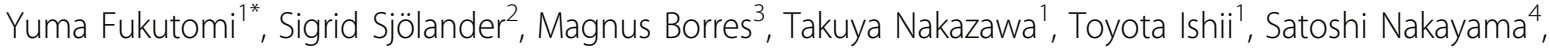 \\ Akira Tanaka ${ }^{4}$, Masami Taniguchi ${ }^{1}$, Akemi Saito ${ }^{1}$, Hiroshi Yasueda ${ }^{1}$, Hiroyuki Nakamura ${ }^{5}$, Kazuo Akiyama ${ }^{1}$
}

From Food Allergy and Anaphylaxis Meeting 2011

Venice, Italy. 17-19 February 2011

\section{Background}

Recent evidence has shown that birch pollen-related soybean allergy mediated by Gly $\mathrm{m} 4$ is common in central Europe. However, the impact of sensitization to Gly $\mathrm{m} 4$ in soybean allergic patients in a population with a low prevalence of Betulaceae pollen allergy and a high soybean consumption is unknown.

\section{Methods}

The aim of this study was to elucidate the prevalence of sensitization to rGly $\mathrm{m} 4$ in adults with soybean allergy, and to analyze the diagnostic efficiency of the IgE antibody to rGly $\mathrm{m} 4\left(\right.$ ImmunoCAP $\left.^{\circledR}\right)$ in soybean allergy in central Japan. Twenty-one soybean-allergic patients were prospectively recruited from Jan. to Dec. 2009, and their levels of IgE antibody to rGly m 4 were compared with those of general alder pollen-allergic control subjects without soybean allergy $(n=85)$.

\section{Results}

Although sensitization to alder pollen was not prevalent in the general outpatients of allergy departments, all the soybean-allergic patients were sensitized to alder pollen and rGly $\mathrm{m}$ 4. Sixty-two percent of the general alder pollen-allergic control subjects were also sensitized to rGly $\mathrm{m} 4$. However, the levels of IgE antibody to rGly $\mathrm{m}$ 4 in soybean-allergic patients were markedly higher than those in alder pollen-allergic control subjects. The area under the receiver-operating characteristics curve for levels of IgE antibody to rGly $\mathrm{m} 4$ in the diagnosis of soybean allergy was 0.86 , which was significantly higher than that to the natural soybean extracts.

\section{Conclusion}

A strong relationship between adult soybean allergy and sensitization to rGly $\mathrm{m} 4$ was also observed in this population with high soybean consumption. The level of IgE antibody to CAP-rGly m 4 was an effective tool in discriminating soybean allergy from general alder pollen-allergy. This result highlights the impact of respiratory allergy to pollen-derived cross-reactive allergens on the epidemics of adult plant food allergy.

\section{Author details}

Sagamihara National Hospital, Clinical Research Center for Allergy and Rheumatology, Sagamihara Kanagawa, Japan. ${ }^{2}$ Phadia AB, Uppsala, Sweden. ${ }^{3}$ Göteborg University, Department of Pediatrics, Sahlgrenska Academy, Göteborg, Sweden. ${ }^{4}$ Phadia KK, Tokyo, Japan. ${ }^{5}$ Graduate School of Medical Science, Kanazawa University, Department of Environmental and Preventive Medicine, Kanazawa Ishikawa, Japan.

Published: 12 August 2011

\section{doi:10.1186/2045-7022-1-S1-P89}

Cite this article as: Fukutomi et al: Soybean allergy in a population with a low prevalence of betulacae pollen allergy and a high soybean consumption. Clinical and Translational Allergy 2011 1(Suppl 1):P89.

'Sagamihara National Hospital, Clinical Research Center for Allergy and

Rheumatology, Sagamihara Kanagawa, Japan

Full list of author information is available at the end of the article 